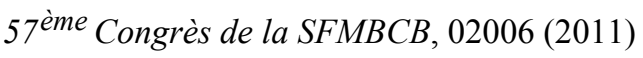

DOI:10.1051/sfmbcb/20115702006

(C) Owned by the authors, published by EDP Sciences, 2011

\title{
Rôle de l'inflammation buccale sur la survenue des AVC ischémiques : le point sur la question
}

\author{
Lafon $\mathrm{A}^{1}$, Lagrost $\mathrm{L}^{2}$, Giroud $\mathrm{M}^{3}$, Ahossi $\mathrm{V}^{1}$, Larras $\mathrm{P}^{1}$, Perrin $\mathrm{D}^{1}$ \\ ${ }^{1}$ Service d'Odontologie, CHU, Dijon, France \\ ${ }^{2}$ Unité INSERM U866, CHU, Dijon, France \\ ${ }^{3}$ Service de Neurologie, CHU, Dijon, France
}

Cette communication propose d'évaluer l'impact réel des foyers infectieux bucco-dentaires dans la survenue des AVC en s'appuyant sur la bibliographie et sur les résultats d'une étude cas/témoins (40 patients avec un AVC versus 40 patients sans AVC) réalisée dans le Service d'Odontologie de Dijon.

L'incidence des AVC d'origine ischémique est en augmentation malgré la prise en charge thérapeutique et préventive des facteurs de risque des AVC; l'athérosclérose représente le principal responsable de la survenue des AVC. L'excès de "mauvais" cholestérol (LDL) joue un rôle important mais il n'est pas le seul à intervenir. D'autres facteurs de risques entrent en jeu dans la formation de la plaque d'athérome : l'hypertension, le surpoids, le diabète, le tabagisme et plus récemment, on a évoqué l'inflammation. En effet, plusieurs études ont mis en évidence le rôle de l'inflammation buccale dans la survenue de l'athérogénèse et des pathologies athéromateuses en général (Mattila et al. 1989, Beck et al. 1996). Il est intéressant de situer le rôle de l'inflammation engendrée par les foyers infectieux buccodentaires dans la survenue des AVC d'autant plus qu'un AVC sur dix reste sans étiologie. Dans la littérature, on ne retrouve que 15 études s'intéressant au rôle de l'inflammation buccale sur la survenue des AVC dont 7 études cas/témoins. Ces études, aux résultats contradictoires, portent uniquement sur les maladies parodontales ou sur le nombre de dents absentes, en omettant les autres foyers infectieux de la cavité buccale. Ces données épidémiologiques ne sont pas suivies d'explications physiopatholgiques. Ceci impute d'autant les possibilités thérapeutiques sur l'apparition, la récidive et la prévention des AVC.

En collaboration avec l'Unité INSERM U866 et le Service de Neurologie du CHU de Dijon, le Service d'Odontologie se propose d'étudier le rôle de l'inflammation buccale sur l'athérogénèse dans la survenue des AVC. Les foyers infectieux buccodentaires ont des répercutions systémiques majeures par l'endotoxémie qu'ils génèrent (Johnson et al. 2005), cette dernière constitue un facteur stimulant la sécrétion de cytokines pro-inflammatoires et pro-athéromateuses. Les premiers résultats confirment l'existence d'une inflammation buccale significativement plus importante chez les patients ayant un AVC. Ils révèlent des taux de CRP ( $\geq$ à $7 \mathrm{mg} / \mathrm{L}$ ) plus élevés chez les patients atteints d'un AVC sans facteurs de risque connus par rapport aux témoins (CRP $\leq$ à $3 \mathrm{mg} / \mathrm{L}$ ) et aux patients ayant un AVC avec au moins un facteur de risque documenté (CRP $\geq 5 \mathrm{mg} / \mathrm{L})$. Ces différences de taux de la CRP, liées en partie au contexte inflammatoire buccal, suggèrent des différences interindividuelles dans la possibilité de détoxification. Nos résultats confirment le rôle du cholestérol (HDL protecteur versus LDL pro-athéromateux) allant dans le sens de l'existence de liens entre le bilan inflammatoire et le bilan lipidique. Ces conclusions ont amené la mise en place, avec le CHR de Metz-Thionville, d'une étude transversale bicentrique pour évaluer une nouvelle piste de détoxification des lipolysaccharides par une lipoprotéine, la PLTP (phospholipid transfer protein).

Ce travail est soutenu par la bourse de la SFMBCB.

Correspondance : arnaud.lafon@chu-dijon.fr 